\title{
A Variable Impedance Power Meter, and Adjust- able Reflection Coefficient Standard
}

\author{
Glenn F. Engen
}

(October 10, 1963)

\begin{abstract}
Most microwave power meters, such as those of the bolometric and calorimetric types, completely absorb the power which they indicate. The use of these meters is thus usually accompanied by the requirement to either (1) determine the power at another place in the microwave circuit from the meter reading, or (2) determine the power which will be delivered to a different load which is to be substituted for the meter.

The evaluation of these relationships plays an important role in the power measurements art.

These problems are alleviated by the power measuring device to be described. This instrument, which is based on reflectometer techniques, may be used either as a feed-through power meter which indicates the power delivered to loads of arbitrary impedance, or as a terminating power meter whose input impedance can be adjusted to arbitrary values without requiring recalibration.

In a slightly modified form the instrument also gives an indication of the reflection coefficient magnitude to which it has been adjusted.

A comprehensive error analysis permits the accuracy to be determined under general operating conditions. 7
\end{abstract}

\section{Background}

Power meters, for the high, ultrahigh, and microwave frequency spectrum, may usually be classed as either "feed-through" or "terminating" types. The former includes a section of appropriate waveguide and contains provision for measuring the power flow, while absorbing as little energy as possible. The second type of meter terminates the waveguide and, ideally, absorbs all of the incident power. That is, the power meter terminates the waveguide by its characteristic impedance. At microwave frequencies, most power meters belong to this second category.

In the use of these power meters, comparison theory and techniques play an important role. This theory deals with predicting the power delivered to a load of arbitrary characteristics when the power absorbed by a different load is known (usually a terminating power meter). In the laboratory, this problem may take the form of calibrating one terminating power meter in terms of a second and similar type meter. In the field it may be required to determine the power delivered to an antenna by the use of a meter of this type. With few exceptions, the use of a terminating type power meter is motivated by the desire to predict the power which will be delivered by a particular signal source to another load whose impedance differs from that of the power meter. Unfortunately, the associated problems are not as widely appreciated as they should be. It is probably safe to say that in many cases the accuracy realized in the current art is to a substantial degree limited by the failure to apply this comparison theory.

The variable impedance power meter, which is the subject of this paper, provides a potential simplification in this comparison theory and procedure because its impedance may be adjusted to equal that of the second meter or load with which it is being compared. In a slightly modified form, this device also provides the magnitude of the reflection coefficient to which it has been adjusted. Because of the strong interdependence between these applications, they will be developed simultaneously, but with major emphasis on the power meter theory. The adjustable reflection coefficient standard will be included as a modification of the power meter application. 


\section{Introduction}

If two loads are connected in turn to a constant voltage, arbitrary impedance, signal source as indicated in figure 1, it can be shown that the ratio of the two powers is given by: [1] ${ }^{1}$

$$
\frac{P_{A}}{P_{B}}=\frac{\left|1-\Gamma_{g} \Gamma_{B}\right|^{2}}{\left|1-\Gamma_{g} \Gamma_{A}\right|^{2}} \frac{1-\left|\Gamma_{A}\right|^{2}}{1-\left|\Gamma_{B}\right|^{2}}
$$

where $P_{A}$ and $P_{B}$ are the powers absorbed by the loads of reflection coefficient $\Gamma_{A}$ and $\Gamma_{B}$, respectively, and $\Gamma_{g}$ is the reflection coefficient of the signal source. A major portion of the comparison theory and techniques, referred to earlier, is devoted to various methods of coping with the relationships and measurement problems implicit in this equation.

The existing techniques for dealing with this problem include the following.

(1) Measurements of $\Gamma_{g}, \Gamma_{A}$, and $\Gamma_{B}$, and substitution of their values in eq (1). This approach is seldom, if ever, used. Note that both the magnitudes and phase angles of the reflection coefficients are required. Although a slotted line is capable of providing both pieces of information, the procedures associated with the phase angle determination are somewhat awkward and the overall accuracy leaves something to be desired. The reflectometer provides a more accurate measurement of the reflection coefficient magnitude, but does not, in its usual form, provide any phase information.

(2) Adjust $\Gamma_{g}=0$ and measure $\left|\Gamma_{A}\right|$ and $\left|\Gamma_{B}\right|$. This is a useful technique when one is interested in comparing two power meters. The techniques for matching a generator include the use of attenuators and load isolators such that the equivalent generator impedance at the terminal surface of interest tends to become independent of the actual source of energy. An alternative equivalent to a matched generator is provided by the use of a directional coupler and associated techniques $[2,3]$.

(3) Use of a "reflectometer type" transfer instrument [4]. The cited reference [4] describes an alternative technique for comparing power meters which utilizes a four-arm waveguide junction together with appropriate power detectors and calibration procedures. This device may be regarded as a feed-through type wattmeter. For example, if a reflectometer is employed as the four-arm junction, it can be shown that the instrument performs the operations required in item 2 implicitly. It should be noted, however, that a reflectometer is only a special case of a more general class of four-arm junctions which satisfy the criteria outlined in [4].

(4) Adjust one of the impedances so that $\mathrm{I}_{A}=\Gamma_{B}$. In this case, the ratio $P_{A} / P_{B}$ reduces to unity. Of the methods described, this one is potentially the most accurate, at least in

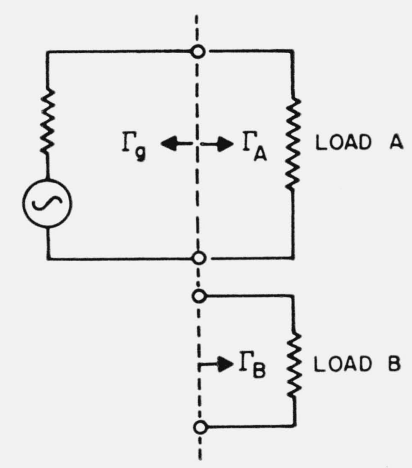

Figure 1. Basic circuit for power measurements by comparison.

\footnotetext{
1 Figures in brackets indicate the literature references at the end of this paper.
} 


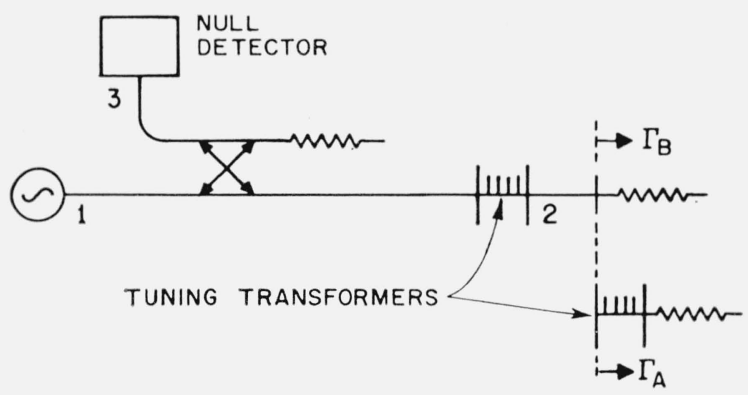

FIGURE 2. Circuit for recognizing the equality of two impedances.

reducing the uncertainty in the ratio $P_{A} / P_{B}$. In addition, while the measurement of ultrahigh frequency and microwave impedance is an exacting art, the techniques for recognizing the equality of two impedances are comparatively simple.

This may be done as indicated in figure 2. For definiteness, it will be assumed that $\Gamma_{A}$ is variable while $\Gamma_{B}$ is fixed in value. The procedure consists of terminating arm 2 of the coupler by $\Gamma_{B}$ and adjusting the transformer for a detector null. Load B is then replaced by load A. If $\Gamma_{A}$ is now adjusted so that a detector null is again realized, $\Gamma_{A}=\Gamma_{B}$ as required.

In the discussion to follow, it will be assumed that load $\mathrm{A}$ is a terminating type power meter of reflection coefficient $\Gamma_{A}$ and which has been calibrated by some means such that it indicates the absorbed power. It will frequently be referred to as the "standard." Load B, for which the power is required, may be another power meter or other termination such as an antenna.

The technique outlined above of making $\Gamma_{A}=\Gamma_{B}$ has found but little use because of the implicit requirement for a tuning transformer, and the uncertainty it can introduce. For example, if this transformer is added ahead of or included as part of the standard, an uncertainty is introduced into its calibration unless the transformer loss is known. Alternatively if the transformer is introduced in conjunction with load B, it must either become part of this load or a similar error will be introduced. Although the attenuation in a typical waveguide transformer is probably not large, the uncertainty in this loss usually precludes the use of transformers in this manner. This technique of adjusting for $\Gamma_{A}=\Gamma_{B}$ has found, however, a limited use at the Boulder Laboratories of the National Bureau of Standards. Here the impedance of a bolometer mount of special design is adjusted, before calibration, to equal that of the load of interest. The design of the NBS bolometer mount permits its calibration in the microcalorimeter [5] and this calibration procedure is independent of the bolometer mount impedance.

The proposed power meter, which is the primary subject of this paper, is of the terminating type and includes a means of adjusting its input impedance without requiring recalibration. In common with other terminating meters, it indicates the net power absorbed. However, the problem of transformer losses is avoided and, within certain limits, this meter may be regarded as the equivalent of a conventional terminating type power meter preceded by a lossless and adjustable tuning transformer.

This device is in appearance, a tuned reflectometer, and in fact an alternative use for the "power transfer instrument" described in [4]. For a simplified explanation, consider figure 3. By use of tuners $T_{x}$ and $T_{y}$ it is possible to compensate for imperfect coupler directivity, etc., so that the powers indicated by detectors 3 and 4 are respectively proportional to the powers associated with the incident and emergent waves on arm 2. These proportionality factors may be determined by a suitable calibration.

If arm 1 is connected to the signal source, the device may be regarded as a feed-through wattmeter since the net power leaving arm 2 is the difference between the emergent and incident powers. Alternatively, with arm 2 connected to the signal source, and arm 1 ter- 


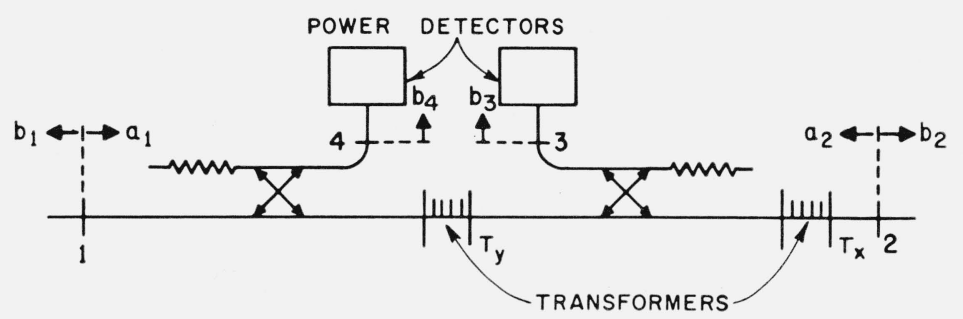

FIGURE 3. Basic four arm junction used in power and impedance measuring applications.

minated by a passive load, the device becomes a terminating power meter with the net power delivered to arm 2 given by the difference between the incident and emergent powers. ${ }^{2}$

The impedance observed at arm 2 under these conditions depends, to some extent, upon the impedance terminating arm 1. On the other hand, the proportionality factors (between the incident or emergent waves at arm 2 and the detectors on arms 3 and 4) are independent of the impedance which terminates arm 1. This makes possible the described operation. In the sections to follow the pertinent theory will be developed. For the sake of completeness, and in order to bring out those features which are pertinent to the present applications, the earlier theory $[3,4,6]$ will be reviewed in some detail.

\section{Review of Pricr Art}

Consider first a four arm waveguide junction assembled from a pair of directional couplers and tuning transformers as shown in figure 3 . Arms 3 and 4 are terminated by constant impedance power detectors. Since the subsequent operation does not require access to the terminal surfaces between the junctions and these detectors, it is not required that the terminal surfaces employed in the analysis coincide with those of the mating, waveguide flanges. This permits a substantial simplification in the treatment by use of the following artifice. For convenience it will be assumed that the power detectors are of the bolometric type. In general the bolometer mount reflects a certain fraction of the incident energy and absorbs another portion. The remainder is delivered to the bolometer or power sensing element. It is thus possible to construct an equivalent circuit of the bolometer mount as shown in figure 4. This circuit consists of a lossless transformer, a matched attenuator, and an "ideal" detector connected in tandem as shown. The characteristics of the transformer and attenuator are adjusted as required to duplicate the power reflected and absorbed ${ }^{3}$ by the actual bolometer mount. The "ideal" detector by definition includes a matched and lossless waveguide structure which delivers all of the incident power to the bolometer element. The power detectors shown in figure 3 are now replaced by these equivalents and the terminal surfaces between the four arm junction and detector chosen as indicated in figure 5. This procedure, in effect, shifts the nonideal behavior of the detectors to the junction, and incorporates it as part of the junction behavior. This stratagem permits an analysis of the general behavior of the device

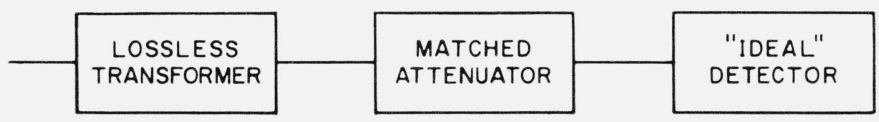

FIGURE 4. Equivalent circuit of bolometer mount (power detector).

2 The reader may wonder if the power input at arm 1 could also be obtained from the readings on arms 3 and 4 . In order to achieve this result the junction would have to satisfy certain additional conditions, the realization of which would prove difficult in the existing art.

${ }^{3}$ Strictly speaking the attenuator is adjusted so that its efficiency equals the effective efficiency of the bolometer mount. These concepts are explained in [5]. 


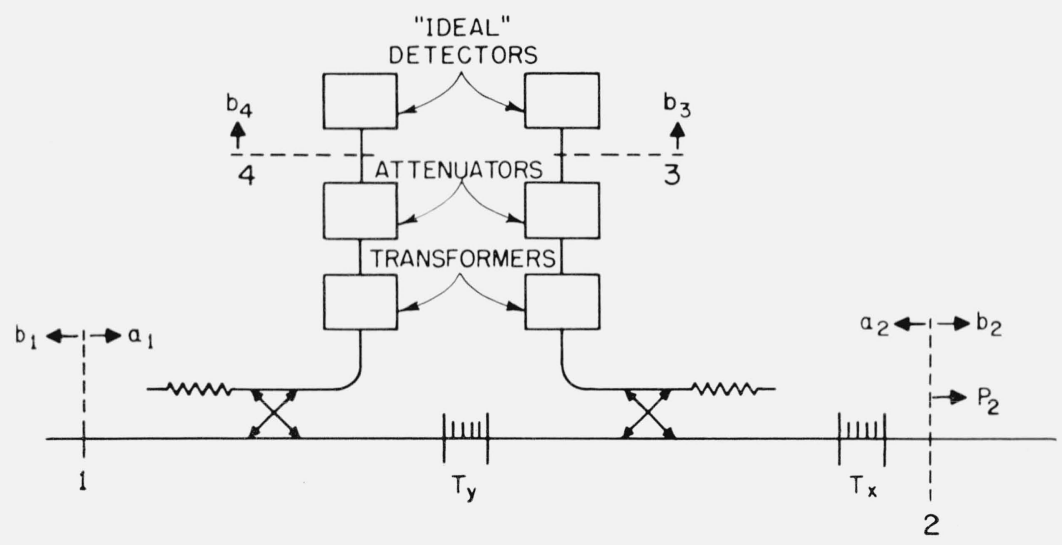

Figure 5. Illustrating the choice of terminal surface employed in the analysis.

under the simplifying assumption of matched and ideal detectors on arms 3 and 4 . It must be remembered, however, that the new terminal surfaces 3 and 4 are, in general, not available for supplementary observations. In addition, certain of the detector characteristics have been absorbed within the junction. These must be taken into account in any attempt to construct the junction behavior from the characteristics of its individual components.

The system behavior will be described in terms of appropriately normalized incident and emergent "voltage" wave amplitudes at the different terminal surfaces. These will be designated by $a_{m}$ and $b_{m}$ respectively, where the subscripts take the values 1 . . 4 corresponding to the different terminals of interest.

The powers, $P_{3}$ and $P_{4}$, delivered to detectors 3 and 4 are given by $\frac{\left|b_{3}\right|^{2}}{Z_{0}}$ and $\frac{\left|b_{4}\right|^{2}}{Z_{0}}$ respectively. (Note that the $a$ 's and $b$ 's are in general complex.) The net power emerging from arm 2 is given by:

$$
P_{2}=\frac{1}{Z_{0}}\left[\left|b_{2}\right|^{2}-\left|a_{2}\right|^{2} \mid\right.
$$

In accordance with frequently used microwave practice, the normalization is made in such a way that the characteristic impedance, $Z_{0}$, reduces to unity. Thus, this factor does not appear in the equations which follow.

The relationships imposed by the junction on the $a$ 's and $b$ 's are given by:

$$
\begin{aligned}
& b_{2}=S_{21} a_{1}+S_{22} a_{2} \\
& b_{3}=S_{31} a_{1}+S_{32} a_{2} \\
& b_{4}=S_{41} a_{1}+S_{42} a_{2}
\end{aligned}
$$

where the $S_{m, n}$ are the scattering coefficients of the four arm junction (a fourth equation involving $b_{1}$ could be written but is not required in the analysis). The advantage of the choice of reference planes outlined earlier is now evident. Ordinarily eqs (3) would include terms in $a_{3}$ and $a_{4}$. Six terms have been eliminated by choosing the terminal surfaces so that $a_{3}$ and $a_{4}$ vanish.

Solving eqs (3) for $a_{2}$ and $b_{2}$ in terms of $b_{3}$ and $b_{4}$ yields:

$$
\begin{aligned}
& a_{2}=\frac{D b_{3}-B b_{4}}{\Delta} \\
& b_{2}=\frac{A b_{4}-C b_{3}}{\Delta}
\end{aligned}
$$


where

$$
\begin{aligned}
& A=S_{21} S_{32}-S_{31} S_{22} \\
& B=S_{31} \\
& C=S_{21} S_{42}-S_{41} S_{22} \\
& D=S_{41} \\
& \Delta=S_{41} S_{32}-S_{31} S_{42} .
\end{aligned}
$$

Substituting eqs (4) into eq (2) gives:

$$
P_{2}=\frac{|A|^{2}-\mid B^{2}}{|\Delta|^{2}}\left|b_{4}\right|^{2}-\frac{|D|^{2}-\mid C^{2}}{|\Delta|^{2}}\left|b_{3}\right|^{2}+\frac{1}{|\Delta|^{2}}\left[\left(B D^{*}-A C^{*}\right) b_{3}^{*} b_{4}+\left(B^{*} D-A^{*} C\right) b_{3} b_{4}^{*}\right]
$$

where $(*)$ denotes the complex conjugate. Equation (5) gives the net power emerging from arm 2 in terms of $b_{3}, b_{4}$ and the parameters of the junction.

For reflectometer applications it is desirable to express $b_{3} / b_{4}$ as a function of $a_{2}$ and $b_{2}$. Equations (4) may be rearranged to yield.

$$
\frac{b_{3}}{b_{4}}=\frac{A \frac{a_{2}}{b_{2}}+B}{C \frac{a_{2}}{b_{2}}+D} .
$$

If arm 2 is terminated by a passive load, of reflection coefficient $\Gamma_{l}$, this becomes:

$$
\frac{b_{3}}{b_{4}}=\frac{A \Gamma_{l}+B}{C \Gamma_{l}+D}
$$

since, under the specified conditions, $a_{2} / b_{2}=\Gamma_{l}$.

Thus far the only explicit assumption concerning the four arm junction is that its behavior is described by eqs (3). (This involves a number of implicit assumptions such as linearity, etc.). By use of the tuners $T_{x}$ and $T_{y}$ it is possible to impose a variety of conditions on the constants $A$. . D. For example, in the reflectometer application, it is required that $B$ and $C$ be made negligibly small as compared with $A$ and $D$, respectively. This may be achieved by the adjustment of $T_{x}$ and $T_{y}$ as described by Engen and Beatty [6].

Note that if both $B$ and $C$ vanish, $b_{3} / b_{4}$ is proportional to $\Gamma_{l}$. In addition,

$$
\begin{gathered}
\left|a_{2}\right|^{2}=\left|\frac{D}{\Delta}\right|^{2}\left|b_{3}\right|^{2}, \quad\left|b_{2}\right|^{2}=\left|\frac{A}{\Delta}\right|^{2}\left|b_{4}\right|^{2}, \\
P_{2}=\left|b_{2}\right|^{2}-\left|a_{2}\right|^{2}=\left|\frac{A}{\Delta}\right|^{2}\left|b_{4}\right|^{2}-\left|\frac{D}{\Delta}\right|^{2}\left|b_{3}\right|^{2},
\end{gathered}
$$

and the powers observed by detectors 3 and 4 are respectively proportional to the powers associated with the incident and emergent waves in arm 2.

Another condition of interest is that in which $B D^{*}-A C^{*}$ is made to vanish. (Note that this is satisfied if $B=C=0$.) In general this may be achieved by the adjustment of $T_{y}[4,6]$ only. In this case eq (5) becomes

where

$$
P_{2}=k_{1}\left|b_{4}\right|^{2}-k_{2}\left|b_{3}\right|^{2}=k_{1} P_{4}-k_{2} P_{3}
$$

$$
\begin{aligned}
& k_{1}=\frac{|A|^{2}-|B|^{2}}{|\Delta|^{2}} \\
& k_{2}=\frac{|D|^{2}-|C|^{2}}{|\Delta|^{2}} .
\end{aligned}
$$

Although eqs (9) and (10) are similar in form, there is an important difference. In the right side of eq (9) the power represented by the two terms may be associated with the incident 
and emergent waves in arm 2. In eq (10), the net power leaving the junction is similarly given by the difference between two terms, but these terms no longer bear a simple relationship to the incident and emergent waves. That is, as a consequence of satisfying only the condition $B D^{*}-A C^{*}=0$ instead of $B=C=0$, it is no longer possible to resolve the "incident" and "emergent" powers at arm 2.

The fact that the net emergent power can be written in the form of eq (10) is important, however, since this provides the basis for proving that the operation is (ideally) independent of impedance discontinuities at terminal surface 2 [4].

\section{General Theory}

In the foregoing section, four arm junction theory has been reviewed and adjustments described which are useful in reflectometer and power measuring applications. The associated calibration procedures have been omitted, but will be described in the following section.

The foregoing analysis has been based on the assumption-implicit or explicit - that the junction will be energized via arm 1 and a passive load connected to arm 2. When used in conjunction with appropriate calibration procedures, to be described, the given techniques permit a determination of the power delivered to a load on arm 2 and/or the magnitude of its reflection coefficient.

However, with the exception of eq (7) the relationships expressed by eqs (2 through 10) are valid for excitation applied via either arm 1 or 2 (or both). The termination of arm 1 by a passive but adjustable load, as shown in figure 6 , permits the same junction to be used in several additional applications, in which the excitation is supplied via arm 2 .

Consider first eq $(10)$. Since $k_{1}$ and $k_{2}$ are functions only of the junction parameters, their values will be independent of the adjustment of transformer $T_{t}$ (fig. 6) which is part of the load terminating arm 1. On the other hand, the impedance "looking into" arm 2 will, in general, depend on this adjustment. Assuming the constants $k_{1}$ and $k_{2}$ have been determined, the measurement of $P_{3}$ and $P_{4}$ thus yields the net input power (at arm 2 ) for arbitrary values of input impedance. (Note that $P_{2}$, eq (10) will now be negative since $P_{2}$ is, by definition, the net emerging power.)

The entire assembly shown in figure 6 may thus be regarded as a terminating type power meter. In the area of power measurement additional applications include:

(1) A variable impedance terminating type power meter. The utility of such an instrument will be evident from the discussion found in the introduction.

(2) A means for measuring the maximum available power from a signal source.

In this latter application the load impedance is adjusted until the maximum power is indicated, ${ }^{4}$ as given by eq $(10)$. Theoretically, the input impedance of the power meter will now be the complex conjugate of the source impedance. In principle, this also provides a means of measuring the impedance of an active source by adjusting for maximum power transfer and then measuring the impedance at arm 2 of the junction. As a practical measure,

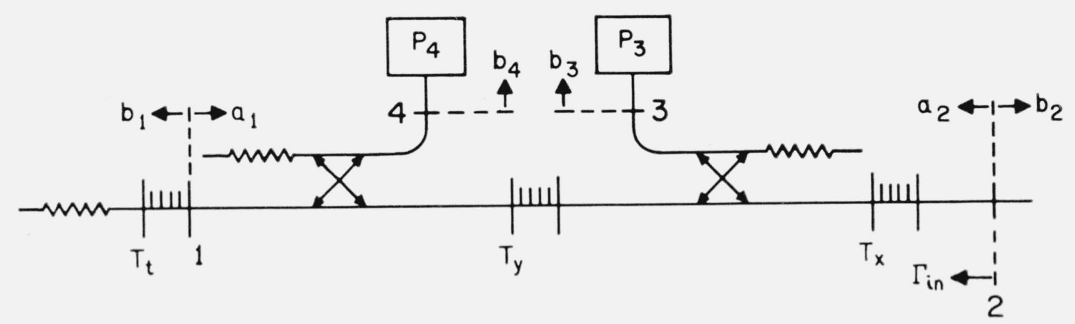

Figure 6. Addition of passive and adjustable load to provide further applications.

${ }^{4}$ The practical utilization of this idea would probably require accessory instrumentation to perform the operations indicated in eq (10). 
un"ortunately, it cannot be necessarily anticipated that this technique will yield a high degree of accuracy because the dependence of power transfer upon load impedance vanishes as the maximum is approached.

For these two applications it is only necessary that the junction satisfy the condition $B D^{*}-A C^{*}=0$.

In impedance measuring applications it is desirable that both $B$ and $C$ vanish. Subject to this latter condition, the reciprocal of eq (6) becomes:

$$
\frac{b_{4}}{b_{3}}=\frac{D}{A} \Gamma_{\text {in }}
$$

where $\Gamma_{\mathrm{ln}}=\frac{b_{2}}{a_{2}}$ is the reflection coefficient "looking into" arm 2 .

Because the indicated detection techniques respond only to the magnitudes of $b_{3}$ and $b_{4}$ it is appropriate to write this equation in the form:

$$
\left|\frac{b_{4}}{b_{3}}\right|=\left|\frac{D}{A}\right|\left|\Gamma_{\text {in }}\right| \cdot
$$

Subject to the determination of $\left|\frac{D}{A}\right|$, the measurement of the ratio of $\left|b_{3}\right|$ to $\left|b_{4}\right|$ thus determines the magnitude of the input reflection coefficient at arm 2 .

There are a number of possible applications for such a device in the field of impedance measurement. Perhaps the most attractive is that of providing a variable impedance for evaluating a lower order accuracy instrument - such as a slotted line.

For completeness, it may be noted that the device could also be used as (1) an impedance transfer standard and (2) to measure unknown reflection coefficient magnitudes. In the latter case, for example, the impedance is first adjusted to equal that of the unknown (by means of the technique described in conjunction with fig. 2), and the reflection coefficient magnitude determined from the indications on arms 3 and 4 . In general, however, these two measurement requirements are more readily satisfied by existing techniques.

Finally, the impedance and power measuring applications may be combined in an application such as measuring the power output of a signal source as a function of load impedance, e.g., a Rieke diagram. For this application, if the phase information is required, certain modifications and extensions of the system are desirable. These details, however, are not within the scope of the present paper.

It should be emphasized that the tuning procedures required for these applications are identical to those employed in the prior art. Thus the cited applications may be regarded as further uses for the existing techniques. For example, the "variable impedance terminating type power meter" is nothing more than the "power transfer instrument" of [4], with an adjustable impedance connected to arm 1 . Thus, with appropriate adjustment and calibration, the same device may be employed as either a feed-through or terminating type power meter.

\section{Calibration Procedures}

The tuning procedures required in the foregoing techniques are described in [6] and need not be repeated here. A similar observation applies to much of the calibration procedure. However, because certain modifications are indicated by these newer applications, this section will also include a review of some of the prior art.

For power measurement applications, the values of $k_{1}$ and $k_{2}$ (eq (10)) are required. As outlined in [4] these may be determined by the following procedure which includes two steps.

(1) Arm 2 is terminated by a standard ${ }^{5}$ power meter, arm 1 is connected to a suitable generator and the values of $P_{2}, P_{3}$, and $P_{4}$ are noted.

${ }^{5}$ I.e., a terminating type power meter whose characteristics have been determined in some manner such that an accurate determination of $P_{2}$ is possible. See reference [5], for example. 
(2) The standard power meter is then replaced by a short circuit $\left(P_{2}=0\right)$ and the corresponding values of $P_{3}$ and $P_{4}$ again noted. Substitution of the appropriate values in eq $(10)$ yields a pair of equations which can be solved for $k_{1}$ and $k_{2}$.

This procedure is based on the fact that eq (10) must hold for all operating conditions. Thus the observation of $P_{3}$ and $P_{4}$ for two known values of $P_{2}$ is, in general, sufficient to determine $k_{1}$ and $k_{2}$.

A third step, which could be employed in place of either of the other two is as follows:

(3) Arm 1 is terminated by a load such that the impedance observed at arm 2 is equal to that of the standard power meter. Arm 2 of the assembly is then connected to a suitable generator and the values of $P_{3}$ and $P_{4}$ noted. The power absorbed by the junction under this condition is determined by connecting the standard meter to the same source. (Note that $P_{2}$ is now negative.)

Thus a third set of values for $P_{2}, P_{3}, P_{4}$ is obtained which may be substituted into eq (10). This latter step is somewhat more complicated than the other two, thus it is appropriate to investigate the relative merits of the different procedures. This question can perhaps be most easily answered by inspection of eq (5).

Equation (5) may be written in the following form,

where

$$
P_{2}=k_{1} P_{4}-k_{2} P_{3}+2 \epsilon \sqrt{P_{3} P_{4}} \cos \theta
$$

$$
\begin{aligned}
& \epsilon=\frac{\left|B D^{*}-A C^{*}\right|}{|\Delta|^{2},} \\
& \theta=\arg \left[\left(B D^{*}-A C^{*}\right) b_{3}^{*} b_{4}\right],
\end{aligned}
$$

and the other quantities have been previously defined.

Because the indicated instrumentation measures magnitudes only, $\theta$ must be regarded as a random variable. In order to secure the desired operation, it is therefore necessary to make $\epsilon$ vanish. In theory this can be done by using a sliding short to terminate arm 2 and adjusting the appropriate transformer until a constant ratio of $P_{3} / P_{4}$ is obtained. This procedure is described in greater detail in the references.

As a practical measure, however, there is a limit to how closely the desired condition may be achieved. In the existing art this is determined primarily by limitations in the ability to recognize the desired condition. Other potential limitations include the tuner resolution and ability of the system to retain the desired adjustment.

The inability to make $\epsilon$ completely vanish affects both the calibration and subsequent use of the device. During the calibration procedure this effect is the equivalent of an uncertainty in the value of $P_{2}$ by the amount $\pm 2 \epsilon \sqrt{P_{3} P_{4}}$. This, in turn, will be reflected as an uncertainty in the calculated values of $k_{1}$ and $k_{2}$. In operation as either a feed-through or terminating type power meter, the value of $P_{2}$ will again be indeterminate by the amount $\pm 2 \epsilon \sqrt{P_{3} P_{4}}$. This is in addition to the uncertainty introduced into the values of $k_{1}$ and $k_{2}$ during the calibration procedure.

A general statement as to the relative merits of the different calibration procedures can now be given. From an inspection of eq (13) it is evident that the error due to a nonzero value of $\epsilon$, in determining $k_{1}$ and $k_{2}$ respectively, can be minimized if the junction is energized in such a way that the conditions, $k_{2} P_{3}<<k_{1} P_{4}$ and $k_{1} P_{4}<<k_{2} P_{3}$ are satisfied, in turn, during the calibration. In practice these criteria are nominally satisfied by steps 1 and 3 .

On the other hand, the relative importance of $k_{1}$ and $k_{2}$ depends upon the subsequent use which is made of the device. In a terminating power meter application, $k_{2}$ is of prime importance while in the feed-through power meter (power transfer standard) the important constant is $k_{1}$.

Thus if only feed-through applications are anticipated, the use of step 1 and either step 2 or 3 is indicated. If "terminating" applications are planned the calibration should include step 3 and either step 1 or?. Where both applications are envisaged, steps 1 and 3 should be 
employed. In those applications where impedance measurements are included, it is the ratio of $k_{1}$ to $k_{2}$ which is important, and this is provided by step 2. Finally, the use of all three steps provides a useful check on the system performance. Thus the choice of calibration procedure depends primarily upon the intended applications. These considerations may be summarized by noting that, for optimum results, the calibration procedure should include those steps which approximate the intended use. Certain aspects of these procedures will be further developed in the section on error analysis.

\section{Practical Considerations}

The degree of flexibility inherent in these techniques is such as to provide for a wide variety of applications. This can perhaps be best illustrated by considering a number of specific examples. For convenience it will be assumed that the detectors terminating arms 3 and 4 are of the bolometric type and operated in conjunction with a bridge type power meter such that the bolometer resistance is maintained at a constant value.

First, consider the junction illustrated in figures 3 and 6 . This is a useful configuration in applications where the power to be measured exceeds, by $10 \mathrm{db}$ or more, the level at which the bolometric detectors operate. In this configuration the values of $k_{1}$ and $k_{2}$ are approximately equal to the coupling ratios in the first and second couplers (numbered from left to right) respectively. For example, if both coupling ratios are $10 \mathrm{db}, k_{1}$ and $k_{2}$ will approximately equal 10. For a $20 \mathrm{db}$ ratio, these values will nominally equal 100 eic. The value of coupling' ratio also determines over what limits the input VSWR may be adjusted in the terminating power meter application. This is primarily determined by the attenuation which exists between arms 1 and 2. For $10 \mathrm{db}$ coupling, the upper limits for the VSWR is approximately 9.

If the power to be measured is of the level at which the bolometric detectors operate (nominally $0.01-10 \mathrm{mw}$ ), smaller values of $k_{1}$ and $k_{2}$ are desirable. These are provided by the alternative configuration, as shown in figure 7 , where the main arm of the first coupler feeds the side arm of the second. If the coupling ratios are equal, and have a value of $10 \mathrm{db}$ or more, the values of $k_{1}$ and $k_{2}$ are approximately unity. This is a useful configuration for intercomparing and calibrating power meters of the bolometric type.

As a feed-through wattmeter, this configuration suffers from a fairly high insertion loss $-10 \mathrm{db}$ or more in the cited example. Thus the practical applications are limited to those in which power loss is a secondary consideration. In a terminating power meter application, the $10 \mathrm{db}$ coupling limits the input VSWR to values of 1.2 and less. As a general rule, a limited VSWR range is associated with an instrument which provides the smaller values of $k_{1}$ and $k_{2}$.

It is also possible to use single coupler arrangements, as shown in figure 8 . In the existing: art it is more difficult to implement this form of the instrument, both because of the limited availability of suitable components and because the tuning procedure is more difficult. On the

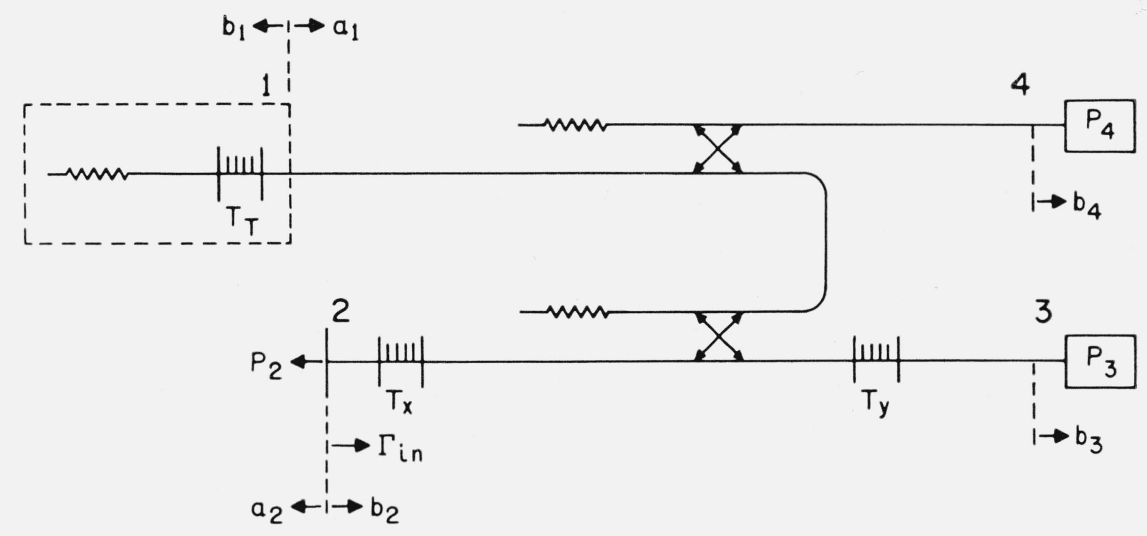

Figure 7. Alternative form of terminating or feedthrough wattmeter. 


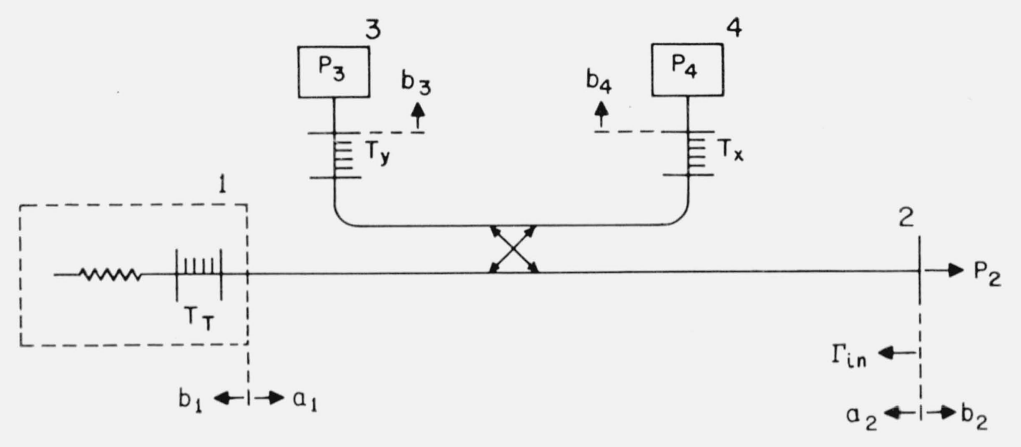

Figure 8. Single coupler form of power meter.

other hand, if a $3 \mathrm{db}$ coupler is employed, this configuration will provide values of 1 and 2 for $k_{1}$ and $k_{2}$ respectively with only $3 \mathrm{db}$ of attenuation, and will permit VSWR's as large as 3 in the terminating application. The single coupler arrangement thus provides a lower combination of values, for $k_{1}, k_{2}$, and insertion loss, than is possible with the two-coupler instruments.

Although bolometric power meters have been specified for arms 3 and 4 , it is evident that any type of constant impedance power detector may be employed. Indeed, absolute power detectors are not actually required. Since only power ratios are involved, these detectors could in principle take the form of a calibrated attenuator followed by a detector which is capable of recognizing when the signal level is at an arbitrary reference value.

In an earlier section it has been noted that, for power measurement applications, it is sufficient that the junction satisfy the condition $B D^{*}-A C^{*}=0$ instead of the condition $B=C=0$ which is required in impedance measuring applications. However, as a practical measure, improved accuracy results if either of the conditions, $k_{1} P_{4}<<k_{2} P_{3}$ or $k_{2} P_{3}<<k_{1} P_{4}$ is satisfied both during the calibration and subsequent operation.

In practical terms this usually means that, when used as a feed-through wattmeter, the VSWR's of the terminating loads (including power meter used for calibration), are limited to values below 1.5. This limit also applies to the input VSWR when employed as a terminating. power meter. In addition, the directivity of the second coupler (including tuner $T_{x}$ if present) should be $20 \mathrm{db}$ or more. In the existing art, these criteria are satisfied by nearly all of the anticipated applications. It is possible, however, to violate the foregoing criteria, and still satisfy the $k_{1} P_{4}<<k_{2} P_{3}$ or $k_{2} P_{3}<<k_{1} P_{4}$ conditions. For example, assume two terminating type power meters are to be compared, and that their impedances, while approximately equal, differ substantially from that required for an impedance match. In this case tuner $T_{x}$ would be adjusted so that $P_{3}$ vanishes when arm 2 is terminated by one of the meters. (This must be done before the adjustment of tuner $T_{y}$.) The condition $k_{2} P_{3}<<k_{1} P_{4}$ is then satisfied.

The preceding paragraphs may be summarized by noting that while the technique provides the means for accurately comparing the power in loads of different impedance, some deterioration in accuracy results if this difference in impedance is large.

\section{Error Analysis}

In the analysis which follows, a number of approximations will be introduced, most of which are based upon the impedance and directivity criteria outlined in the immediately preceding paragraphs. Although, in many instances, the ultimate conclusions are substantially independent of these criteria, in careful work it would be desirable to check the validity of these approximations in an application where there is a marked departure from the stated conditions.

Equation (13) gives the net power flow, $P_{2}$, across the terminal surface of arm 2 (figs. 3, $6,7,8)$, in terms of the powers $P_{3}, P_{4}$, and the junction parameters $k_{1}, k_{2}, \epsilon$, and $\theta$. In general, the error in $P_{2}$ will include a contribution from the uncertainty in each of these quantities. 
Let the uncertainty in $P_{3}$ be denoted by $\delta P_{3}$, etc. Then the limit of error due to the uncertainty in $P_{3}, P_{4}, k_{1}, k_{2}$ and from neglecting $\epsilon$ is given by:

$$
\left|\frac{\delta P_{2}}{P_{2}}\right|=\frac{k_{1} P_{4}\left(\left|\frac{\delta k_{1}}{k_{1}}\right|+\left|\frac{\delta P_{4}}{P_{4}}\right|\right)+k_{2} P_{3}\left(\left|\frac{\delta k_{2}}{k_{2}}\right|+\left|\frac{\delta P_{3}}{P_{3}}\right|\right)+2 \epsilon \sqrt{P_{3} P_{4}}}{\left|k_{1} P_{4}-k_{2} P_{3}\right|} .
$$

Note that in order to determine the maximum error $\theta$ has been chosen so that $|\cos \theta|=1$. The error determination thus reduces to an evaluation of $\epsilon, \delta k_{1} / k_{1}, \delta k_{2} / k_{2}, \delta P_{3} / P_{3}, \delta P_{4} / P_{4}$. These will be discussed in the order listed.

\subsection{Evaluation of $\epsilon$}

As already noted, it is an objective of the tuning procedure to make $\epsilon$ vanish. In theory this can be accomplished by the use of tuner $T_{y}$. The tuning procedure consists of terminating arm 2 by a sliding short and adjusting for a constant ratio, $P_{3} / P_{4}$, for all positions of the short. It is possible to relate the value of $\epsilon$ to the inability to completely realize this condition through the following argument.

The operation of the sliding short may be described by the equation $\Gamma_{2}=e^{i \phi}$ where the value of $\phi$ is determined by the short position. Substitution into eq (7) yields:

$$
\frac{b_{3}}{b_{4}}=\frac{A e^{i \phi}+B}{C e^{i \phi}+D}
$$

It can be shown ${ }^{6}$ that the locus of $b_{3} / b_{4}$, which is generated by varying $\phi$, is a circle of radius

$$
R=\frac{|A D-B C|}{|D|^{2}-|C|^{2}}
$$

The distance from the origin to the center of this circle is given by

$$
R_{c}=\frac{\left|B D^{*}-A C^{*}\right|}{|D|^{2}-|C|^{2}}
$$

As $\phi$ varies, the ratio $\left|b_{3} / b_{4}\right|$ will, in general, take on different values with the maximum and minimum values given by:

$$
\begin{aligned}
& \left|\frac{b_{3}}{b_{4}}\right|_{\max }=R+R_{c} \\
& \left|\frac{b_{3}}{b_{4}}\right|_{\min }=R-R_{c} .
\end{aligned}
$$

This is illustrated in figure 9.

By performing the indicated operations one obtains the result:

In practice, however, it is

$$
\frac{\left|\frac{b_{3}}{b_{4}}\right|_{\max }-\left|\frac{b_{3}}{b_{4}}\right|_{\min }}{\left|\frac{b_{3}}{b_{4}}\right|_{\max }+\left|\frac{b_{3}}{b_{4}}\right|_{\min }}=\frac{R_{c}}{R}=\frac{\left|B D^{*}-A C^{*}\right|}{|A D-B C|} .
$$

$$
\left|\frac{b_{3}}{b_{4}}\right|^{2}=\frac{P_{3}}{P_{4}}
$$

${ }^{6}$ See a text on complex variable theory. 


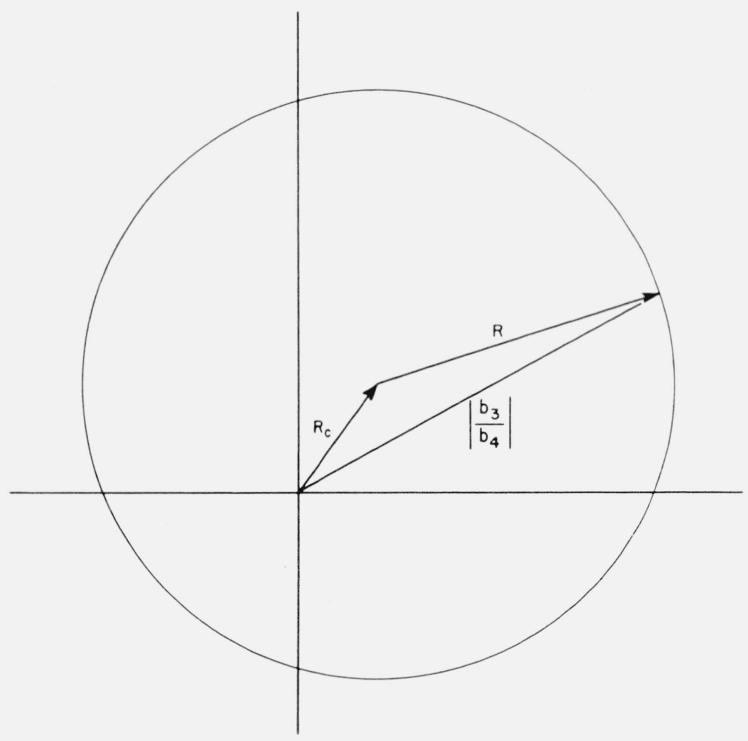

Figure 9. Diagram giving relationship between $\mathrm{R}, \mathrm{R}_{\mathrm{c}}$, and $\left|\frac{\mathrm{b}_{3}}{\mathrm{~b}_{4}}\right|$.

which is observed. If the maximum and minimum values are approximately equal it can be easily shown that:

Let

$$
\frac{\left(\frac{P_{3}}{P_{4}}\right)_{\max }-\left(\frac{P_{3}}{P_{4}}\right)_{\min }}{4\left(\frac{P_{3}}{P_{4}}\right)_{\mathrm{av}}} \approx \frac{\left|\frac{b_{3}}{b_{4}}\right|_{\max }-\left|\frac{b_{3}}{b_{4}}\right|_{\min }}{\left|\frac{b_{3}}{b_{4}}\right|_{\max }+\left|\frac{b_{3}}{b_{4}}\right|_{\min }}
$$

$$
\rho=\frac{\left(\frac{P_{3}}{P_{4}}\right)_{\max }-\left(\frac{P_{3}}{P_{4}}\right)_{\min }}{\left(\frac{P_{3}}{P_{4}}\right)_{\mathrm{av}}}
$$

then

$$
\frac{\left|B D^{*}-A C^{*}\right|}{|A D-B C|}=\frac{\left|B D^{*}-A C^{*}\right|}{|A D| \cdot\left|1-\frac{B C}{A D}\right|}=\frac{\rho}{4} .
$$

Now, by definition,

$$
\frac{\epsilon}{\sqrt{k_{1} k_{2}}}=\frac{\left|B D^{*}-A C^{*}\right|}{|A D|\left(1-\left|\frac{B}{A}\right|^{2}\right)^{1 / 2}\left(1-\left|\frac{C}{D}\right|^{2}\right)^{1 / 2}}
$$

and by hypothesis $\left|B D^{*}-A C^{*}\right|$ is small so that $\frac{B}{A} \approx \frac{C^{*}}{D^{*}}$. Therefore,

$$
\left|\frac{B}{A}\right|^{2} \approx \frac{B C}{A D} \approx\left|\frac{C}{D}\right|^{2}
$$

Substitution of eq (20) into eqs (18) and (19) yields:

$$
\epsilon \approx \frac{\sqrt{k_{1} k_{2}}}{4} \rho
$$

Equation (21) thus gives $\epsilon$ in terms of the measurable quantities $\rho, k_{1}$, and $k_{2}$. 


\subsection{Evaluation of Error Limits for $k_{1}$ and $k_{2}$}

The experimental error, in determining $k_{1}$ and $k_{2}$, may be divided into contributions from two sources: (1) the error due to a nonzero $\epsilon$, and (2) the error which accrues from the uncertainty in the associated values of $P_{2}, P_{3}$, and $P_{4}$. The error in $k_{1}$ and $k_{2}$ will also depend, to a considerable degree, upon which two of the three possible calibration procedures are employed.

In order to include the various possibilities, it is useful to outline the investigation in the following manner.

The two steps employed in the calibration procedure will be designated as "step $a$ " and "step $b . "$

Step $a$ may be either of steps 1 and 2 as listed in section 5 , but not step 3 .

Step $b$ may be either of steps 2 and 3 but not step 1 .

Steps $a$ and $b$ may not represent step 2 simultaneously.

It should be noted that the introduction of these definitions involves no loss in generality. In the equations which follow, the subscripts " $a$ " and " $b$ " will be employed. For example, $P_{3 b}$ represents the power absorbed by the detector on arm 3 during step $b$ of the calibration procedure etc.

\section{a. Error in $k_{1}$ Due to $\epsilon$}

Subject to these definitions, the following equation is obtained for $k_{1}$ :

$$
k_{1}=\frac{P_{2 b} P_{3 a}-P_{2 a} P_{3 b}+2 \epsilon\left(\sqrt{P_{3 a} P_{4 a}} P_{3 b} \cos \theta_{a}-\sqrt{P_{3 b} P_{4 b}} P_{3 a} \cos \theta_{b}\right)}{P_{3 a} P_{4 b}-P_{3 b} P_{4 a}} .
$$

The limit of error, $E_{1 \epsilon}$, due to $\epsilon$ is thus given by:

$$
E_{1 \epsilon}=\frac{2 \epsilon\left[\sqrt{P_{3 a} P_{4 a}} P_{3 b}+\sqrt{P_{3 b} P_{4 b}} P_{3 a}\right]}{P_{2 a} P_{3 b}-P_{2 b} P_{3 a}} .
$$

Use of the approximations,

and rearranging yields:

$$
\begin{aligned}
& P_{2 a} \approx k_{1} P_{4 a}-k_{2} P_{3 a} \\
& P_{2 b} \approx k_{1} P_{4 b}-k_{2} P_{3 b}
\end{aligned}
$$

$$
\begin{aligned}
& E_{1 \epsilon} \approx \frac{2 \epsilon}{k_{1}} \sqrt{\frac{P_{3 a}}{P_{4 a}}} \frac{1+\sqrt{\frac{P_{3 a} P_{4 b}}{P_{4 a} P_{3 b}}}}{1-\frac{P_{3 a} P_{4 b}}{P_{4 a} P_{3 b}}} \\
& E_{1 \epsilon} \approx \frac{\rho}{2} \sqrt{\frac{k_{2} P_{3 a}}{k_{1} P_{4 a}}}\left(1+\sqrt{\frac{P_{3 a} P_{4 b}}{P_{4 a} P_{3 b}}}\right),
\end{aligned}
$$

since in general,

$$
\frac{P_{3 a} P_{4 b}}{P_{4 a} P_{3 b}}<<1
$$

b. Error in $k_{1}$ Due to $P_{2}, P_{3}, P_{4}$

If the terms in $\epsilon$ are ignored, eq (22) may be written:

$$
k_{1}=\frac{\frac{P_{2 a}}{P_{4 a}}-\frac{P_{3 a} P_{2 b}}{P_{4 a} P_{3 b}}}{1-\frac{P_{3 a} P_{4 b}}{P_{4 a} P_{3 b}}} .
$$

When step 2 is included in the calibration procedure, one of the numerator terms must, ideally, vanish since either $P_{2 a}=0$ or $P_{2 b}=0$. Then, because the denominator is essentially equal to 
unity, the experimental error in $k_{1}$ is primarily that in the remaining numerator term.

If the calibration is effected by means of steps 1 and 3 , the conditions

$$
\begin{aligned}
& k_{2} P_{3 a}<<k_{1} P_{4 a} \\
& k_{1} P_{4 b}<<k_{2} P_{3 b}
\end{aligned}
$$

are usually satisfied. In this case it can be easily shown that

$$
\frac{P_{2 a}}{P_{4 a}}>>\frac{P_{3 a} P_{2 b}}{P_{4 a} P_{3 b}}
$$

and the error in $k_{1}$ is essentially that in $P_{2 a} / P_{4 a}$.

The limit of error in $P_{2 a} / P_{4 a}$ will be the sum of the errors in the individual terms. The term $P_{2 a}$ is usually determined by a "standard" power meter and the errors will be of both random and systematic types such as those due to instability and uncertainty in calibration. Conversely, the errors in $P_{4 a}$ will be only of the random type, since any systematic error may be included in the definition of $k_{1}$. Thus when $k_{1}$ is determined by step 1 and either of steps 2 or 3 the limiting uncertainty, $E_{p}$ in $k_{1}$ (due to errors in $P_{2}, P_{3}, P_{4}$ ), may be written:

$$
E_{1 p}=\frac{\left|\delta P_{2 a}\right|}{P_{2 a}}+\frac{\left|\delta P_{4 a}\right|}{P_{4 a}}
$$

In the case where $k_{1}$ is to be determined by the use of steps 2 and $3, k_{1}$ is given by the equation:

$$
k_{1}=\frac{-\frac{P_{3 a} P_{2 b}}{P_{4 a} P_{3 b}}}{1-\frac{P_{3 a} P_{4 b}}{P_{4 a} P_{3 b}}} .
$$

Again, the error is determined primarily by the numerator. But now there are three terms which are subject to random error, and one, $P_{2 b}$, in which the errors are both random and systematic. This expression is also subject to another type of error because the condition, $P_{2 a}=0$, which leads to its use, is never completely satisfied. ${ }^{7}$ This is due to the inability to construct a perfectly reflecting short. For the sake of brevity, an intuitive rather than analytical treatment of this error will be given.

Consider eq (26). The value of this expression must remain invariant when the perfect "short" is replaced by an imperfect one. Thus the change from a zero to nonzero value for the first numerator term must be reflected by a corresponding, and very nearly equal, change in the second term. This change will occur in, and only in, the factors $P_{3 a}$ and $P_{4 a}$ since the factors $P_{2 b}, P_{3 b}$ pertain to the other part of the calibration procedure. Now the ratio $P_{3 a} / P_{4 a}$ is approximately ${ }^{8}$ proportional to $\left|\Gamma_{s}\right|^{2}$, where $\Gamma_{s}$ is the reflection coefficient of the short. Thus it is appropriate to replace eq $(28)$ by:

$$
k_{1}=-\frac{\frac{1}{\left.\Gamma_{s}\right|^{2}} \frac{P_{3 a} P_{2 b}}{P_{4 a} P_{3 b}}}{1-\frac{P_{3 a} P_{4 b}}{P_{4 a} P_{3 b}}} .
$$

The limit of error in $k_{1}$ for this case is thus:

$$
E_{1 p}=\left|\frac{\delta P_{2 b}}{P_{2 b}}\right|+\frac{\left|\delta P_{3 b}\right|}{P_{3 b}}+\frac{\left|\delta P_{3 a}\right|}{P_{3 a}}+\frac{\left|\delta P_{4 a}\right|}{P_{4 a}}+\frac{\left.|\delta| \Gamma_{s}\right|^{2} \mid}{\left|\Gamma_{s}\right|^{2}} .
$$

\footnotetext{
7 The inability to satisfy this condition also results in an error when step 1 is included in the calibration procedure. In this case, however, the contribution from this source is negligible.

${ }^{8}$ This relationship is exact if $B=C=0$.
} 
Comparison with eq (27) indicates that the expected error in a determination of $k_{1}$, using steps 2 and 3 , is considerably in excess of that which results when step 1 is included in the procedure. This bears out the earlier statement concerning the desirability of including in the calibration procedure those steps which correspond to the intended usage. (Note that if only terminating applications are planned, the lower accuracy in $k_{1}$ is not too important because $k_{1} P_{4}$ is typically no more than a few percent of $k_{2} P_{3}$.)

In summary, the uncertainty in $k_{1},\left|\delta k_{1}\right| / k_{1}$, is given by:

$$
\frac{\left|\delta k_{1}\right|}{k_{1}}=E_{1 \epsilon}+E_{1 p}
$$

where

$$
\begin{gathered}
E_{1 \epsilon}=\frac{\rho}{2} \sqrt{\frac{k_{2} P_{3 a}}{k_{1} P_{4 a}}}\left(1+\sqrt{\frac{P_{3 a} P_{4 b}}{P_{4 a} P_{3 b}}}+\text { higher order terms in } \sqrt{\frac{P_{3 a} P_{4 b}}{P_{4 a} P_{3 b}}}\right) \\
E_{1 p}=\frac{\left|\delta P_{2 a}\right|}{P_{2 a}}+\frac{\left|\delta P_{4 a}\right|}{P_{\zeta a}}
\end{gathered}
$$

using steps 1 and 2 or steps 1 and 3 ;

$$
E_{1 p}=\frac{\delta P_{2 b}}{P_{2 b}} \mid+\frac{\left|\delta P_{3 b}\right|}{P_{3 b}}+\frac{\left|\delta P_{3 a}\right|}{P_{3 a}}+\frac{\left|\delta P_{4 a}\right|}{P_{4 a}}+\frac{\left.|\delta| \Gamma_{s}\right|^{2} \mid}{\left|\Gamma_{s}\right|^{2}}
$$

using steps 2 and 3 ; and

$$
\rho=\frac{\left(\frac{P_{3}}{P_{4}}\right)_{\max }-\left(\frac{P_{3}}{P_{4}}\right)_{\min }}{\left(\frac{P_{3}}{P_{4}}\right)_{\mathrm{av}}} .
$$

In an entirely analogous manner it can be shown that the maximum error in $k_{2},\left|\delta k_{2}\right| / k_{2}$, is given by:

where

$$
\frac{\left|\delta k_{2}\right|}{k_{2}}=E_{2 \epsilon}+E_{2 p}
$$

$$
\begin{gathered}
E_{2 \epsilon}=\frac{\rho}{2} \sqrt{\frac{k_{1} P_{4 b}}{k_{2} P_{3 b}}}\left(1+\sqrt{\frac{P_{3 a} P_{4 b}}{P_{4 a} P_{3 b}}}+\text { higher order terms in } \sqrt{\frac{P_{3 a} P_{4 b}}{P_{4 a} P_{3 b}}}\right) \\
E_{2 p}=\left|\frac{\delta P_{2 b}}{P_{2 b}}\right|+\frac{\left|\delta P_{3 b}\right|}{P_{3 b}}
\end{gathered}
$$

using steps 1 and 3 or steps 2 and 3 ; and

$$
E_{2 p}=\frac{\left|\delta P_{2 a}\right|}{P_{2 a}}+\frac{\left|\delta P_{4 a}\right|}{P_{4 a}}+\frac{\left|\delta P_{4 b}\right|}{P_{4 b}}+\frac{\left|\delta P_{3 b}\right|}{P_{3 b}}+\frac{\left.|\delta| \Gamma_{s}\right|^{2} \mid}{\left|\Gamma_{s}\right|^{2}}
$$

using steps 1 and 2 .

$$
\text { 7.3. Evaluation of } \frac{\left|\delta P_{3}\right|}{P_{3}} \text { and } \frac{\left|\delta P_{4}\right|}{P_{4}}
$$

The terms $\left|\delta P_{3}\right| / P_{3}$ and $\left|\delta P_{4}\right| / P_{4}$ (eq (14)) represent the random errors in $P_{3}, P_{4}$ during the measurement procedure. These errors will in general be equal, but in addition to, the errors $\delta P_{3 a} \mid / P_{3 a}$ etc., observed during the the calibration.

\subsection{Summary of Frrors}

By use of the preceding theory it is thus possible to set limits to the error terms introduced in eq (14). The necessary relations are summarized in the preceding paragraph and by eos (17), (21), (27), (30), (31), (32), (33), (34), (35), and (36). 
Finally, if the recommendations as to choice of calibration procedures are followed, and the conditions $|B| \approx|C| \approx 0$ are satisfied, these relations for both applications may be summarized by the approximate formula:

$$
\frac{\left|\delta P_{2}\right|}{P_{2}} \approx \frac{\rho}{2}\left(\left|\Gamma_{c}\right|+\left|\Gamma_{m}\right|\right)+\frac{\left|\delta P_{2 s}\right|}{P_{2 s}}+\frac{\left|\delta P_{c}\right|}{P_{c}}+\frac{\left|\delta P_{m}\right|}{P_{m}}
$$

where the following definitions apply:

$\Gamma_{c}=$ reflection coefficient of power standard employed in calibration procedure,

$\Gamma_{m}=$ reflection coefficient of load in feed-through application, or input reflection coefficient of device in terminating application,

$\frac{\left|\delta P_{2 s}\right|}{P_{2 s}}=$ error in "standard" power meter employed for calibration,

$\frac{\left|\delta P_{c}\right|}{P_{c}}=$ random error in detector $P_{4}$ in feed-through application, or $P_{3}$ in terminating application, during calibration procedure,

$\frac{\left|\delta P_{m}\right|}{P_{m}}=$ as ahove except during measurement procedure.

In many cases the errors

$$
\frac{\left|\delta P_{c}\right|}{P_{c}} \text { and } \frac{\left|\delta P_{m}\right|}{P_{m}}
$$

will be nominally equal. However, if the application calls for a significant difference in power levels during the calibration and measurement procedures, there may be some difference in these terms.

Equation (37) is a convenient "rule of thumb" for many of the anticipated applications. However, where more precise limits of error are required, the more exact equations should be employed.

This error analysis has been limited to the power measurement application. A similar analysis for the adjustable reflection coefficient application is beyond the scope of this paper although it may be anticipated that this would be a comparatively simple extension of the "conventiona!" reflectometer error analysis. [6, 7]

\section{Simmary}

The preceeding sections have given the theoretical and practical details for an instrument which, when properly adjusted and calibrated, can be used as either a feed-through, or variable impedance terminating type power meter. As a feed-through device, the net power to a load of arbitrary impedance is obtained. In the terminating application, the net power absorbed is indicated and this is independent of the input impedance value to which it has been adjusted.

Impedance measurements and mismatch error corrections represent a burdensome and annoying aspect of the current power measurements art. As noted earlier, it is reasonably safe to say that the failure to apply, or ignorance of, these corrections represents a major source of error.

The techniques presented and developed in this paper contain the potential for a significant improvement: both in terms of simplifying the measurement procedure and providing improved accuracy.

In the existing art, however, there are a number of practical problems associated with the implementations of these ideas, such as the time consuming nature of the tuner adjustments. Thus there is room for a considerable amount of developmental work in the reduction of these ideas to practice both in the areas of microwave hardware and associated instrumentation. The important point is that it is now possible (in principle) to construct a microwave power meter which all but eliminates the need for these awkward mismatch error measurements and corrections. 


\section{References}

1] R. W. Beatty and A. C. Macpherson, Mismatch errors in microwave power measurements, Proc. IRE 41, 1112-19 (Sept. 1953).

[2] G. F. Engen, Amplitude stabilization of a microwave signal source, IRE Trans. MTT, MTT-6, 202-06 (April 1958).

[3] G. F. Engen, Recent developments in the field of microwave power measurements at the NBS, IRE Trans. Instr. I-7, 304-06 (Dec. 1958).

[4] G. F. Engen, A transfer instrument for the intercomparison of microwave power meters, IRE Trans. Instr. I-9, 202-08 (Sept. 1960).

[5] G. F. Engen, A refined X-band microwave microcalorimeter, J. Res. NBS, 63C (Engr. and Instr.) No. 1, 77-82 (July-Sept. 1959).

[6] G. F. Engen and R. W. Beatty, Microwave reflectometer techniques, IRE Trans. on Microwave Theory and Techniques, MTT-7, 351-55 (July 1959).

[7] W. J. Anson, A guide to the use of the modified reflectometer technique of VSWR measurement, J. Res. NBS, 65C (Engr. and Instr.) No. 4, 217-223 (Oct.-Dec. 1961).

(Paper 68C1-148) 